\title{
Clinical and Molecular Characterization of S1118F-CFTR
}

\author{
Himabindu Penmatsa, MS ${ }^{1}$, Carla A. Frederick, MD $^{2}$, Sunitha Nekkalapu, $\mathbf{M S}^{1}$, Veronica G. \\ Conoley, BS ${ }^{1}$, Weiqiang Zhang, $\mathrm{PhD}^{1}$, Chunying $\mathrm{Li}^{1}, \mathrm{PhD}^{1,2,3}$, John Kappes, $\mathrm{PhD}^{4}$, Dennis \\ C. Stokes, MD ${ }^{2}$, and Anjaparavanda P. Naren, PhD $^{1,{ }^{*}}$ \\ ${ }^{1}$ Department of Physiology, University of Tennessee Health Science Center, Memphis, Tennessee, \\ USA. \\ 2University of Tennessee Cystic Fibrosis Care and Research Center at Le Bonheur Children's \\ Medical Center, Memphis, Tennessee, USA. \\ ${ }^{4}$ Department of Medicine, University of Alabama at Birmingham, Birmingham, Alabama, USA.
}

\section{Summary}

Cystic Fibrosis is a lethal autosomal recessive disorder usually associated with lung disease, pancreatic insufficiency and high sweat chloride levels.

Clinical case-A patient admitted to Le Bonheur Children's Medical Center (LBCMC, Memphis, TN, USA) showed symptoms of meconium ileus which required exploratory laparotomy, bowel resection and ileostomy. Genotyping showed $\Delta \mathrm{F} 508 / \mathrm{I} 1027 \mathrm{~T}$ on one chromosome and S1118F on the other. Sweat testing on three different occasions gave negative and intermediate results $(22.7,24.6$ $\mathrm{mmol} / \mathrm{L} ; 55.1,58.6 \mathrm{mmol} / \mathrm{L}$ and $55.1,58 \mathrm{mmol} / \mathrm{L}$ ) and pancreatic elastase testing showed normal levels.

Objective-To characterize S1118F-CFTR mutation at a molecular level to help understand the associated CF-phenotype.

Methods-Molecular characterization of S1118F-CFTR mutant was studied in HEK-293 cells at $37^{\circ} \mathrm{C}$. Various biochemical methods such as Western blotting, real-time PCR, Pulse chase labeling and iodide efflux assay were employed.

Results-S1118F-CFTR makes less than 10-15\% of mature CFTR (Band C) compared to WTCFTR. The mRNA levels of S1118F-CFTR and WT-CFTR are comparable. S1118F-CFTR is functional but shows about 10-15\% of WT-CFTR activity. S1118F-CFTR shows impaired maturation and CF-correctors can increase the amount of mature and functional CFTR by 3 to 4 fold.

Conclusion-S1118F-CFTR shows impaired maturation and an individual with S1118F-CFTR paired with $\triangle$ F508-CFTR exhibits atypical CF symptoms with intermediate sweat chloride level and meconium ileus despite documented pancreatic sufficiency.

\section{Keywords}

CFTR mutations; Cystic Fibrosis; impaired maturation; CF-correctors

\footnotetext{
*Correspondence to: Anjaparavanda P Naren, PhD, Associate Professor, Department of Physiology, University of Tennessee Health Science Center, 894 Union Avenue, 426 Nash Building, Memphis, TN 38163, USA. Tel: 901-448-3137, Fax: 901-448-7126, anaren@utmem.edu.

${ }^{3}$ Present address: Department of Biochemistry and Molecular Biology, Wayne State University, Detroit, Michigan, USA.

Presented in part at the 22nd Annual North American Cystic Fibrosis Conference. Orlando, Florida, October 23-25, 2008.
} 


\section{INTRODUCTION}

Cystic Fibrosis Transmembrane Conductance Regulator (CFTR) is a chloride $\left(\mathrm{Cl}^{-}\right)$channel expressed in the epithelial cells of lung, pancreas, gut etc. CFTR gene encodes a 1480 amino acid chloride channel protein with twelve putative transmembrane domains (TMDs), two nucleotide binding domains (NBDs) and a regulatory domain (R domain). ${ }^{1}$ Mutations in CFTR gene cause the expression of abnormal or non-functional chloride channel in most cases. Altered or loss of CFTR chloride channel activity causes the disease Cystic Fibrosis (CF). Cystic Fibrosis is a lethal autosomal recessive disorder generally associated with lung disease, pancreatic insufficiency and high sweat chloride levels. Obstruction of the intestine by thick meconium (meconium ileus) is the most common neonatal presentation for $\mathrm{CF}$, and most cases of meconium ileus are associated with pancreatic insufficiency. ${ }^{-} 3$

Although there are more than 1500 mutations described for the CFTR gene, mutations in CF patients can be divided into six classes. The class I mutations constitute nonsense, splice and frame shift mutants that encode truncated forms of CFTR (e.g., G542X and W1242X). These premature stop mutations are found in $\sim 5 \%$ of CF patients. The class II mutations are mostly processing mutants that get trapped in the endoplasmic reticulum (ER). $\triangle$ F508-CFTR is the most prevalent class II mutant. The class III (regulation mutants) and class IV (permeation mutants) are mutants with partial loss of function. The class $\mathrm{V}$ mutations show reduced mRNA stability. Class VI CFTR mutations have been proposed by the laboratory of GL Lukacs. This class of mutations includes C-terminal truncated CFTR which form unstable mature protein with 5 - to 6 -fold faster degradation rate than WT-CFTR. ${ }^{2-7}$

We present herein a clinical case of a CF patient with intermediate sweat chloride levels, pancreatic sufficiency, meconium ileus and mutations in the CFTR gene $(\triangle \mathrm{F} 508$ and S1118F). The objective of this study is to characterize S1118F-CFTR mutation at protein level to help understand the associated CF-phenotype. The study will have significant implication for future development of mutation specific therapies aimed at restoring CFTR function.

\section{MATERIALS AND METHODS}

Genotyping

Genotyping was performed at Ambry Genetics (CA, USA) which showed $\Delta$ F508/I1027T on one chromosome and S1118F on the other.

\section{Tissue Culture and Site-directed Mutagenesis}

HEK-293 cell line was used to express both wild type (WT) and mutant CFTR. The cells were cultured in DMEM-F12 media (Invitrogen) containing 10\% serum and 1\% Penicillin/ Streptomycin and maintained in $5 \% \mathrm{CO}_{2}$ incubator at $37^{\circ} \mathrm{C}$.

Site-directed mutagenesis was performed by a single point mutation in eukaryotic expression vector (pcDNA3) containing WT-CFTR cDNA using Quikchange site-directed mutagenesis kit (Stratagene) and appropriate primers to make S1118F-CFTR mutant. Lipofectamine 2000 (Invitrogen) was used to transiently transfect HEK-293 cells with pcDNA3 containing WT or mutant CFTR cDNA. ${ }^{8}$

\section{Western Blotting}

Cells transfected with WT or mutant CFTR cDNA were lysed using lysis buffer $(1 \times$ PBS, containing $0.2 \%$ Triton-X-100 and protease inhibitors phenylmethylsulfonyl fluoride $1 \mathrm{mM}$, pepstatin A $1 \mu \mathrm{g} / \mathrm{ml}$, leupeptin $1 \mu \mathrm{g} / \mathrm{ml}$, aprotinin $1 \mu \mathrm{g} / \mathrm{ml}$ ). The lysate was centrifuged at 16,000 $g$ for $10 \mathrm{~min}$ at $4{ }^{\circ} \mathrm{C}$ and the clear supernatant was subjected to immunoprecipitation using $\alpha$ 
24-1 IgG mab (R\&D Systems) crosslinked to protein A/G beads (Santa Cruz). The immunoprecipitated beads were washed three times with lysis buffer and subjected to SDSPAGE on 5\% gel (Bio-rad). The protein was transferred to PVDF membrane and probed for CFTR using $\alpha$ 24-1 mab antibody $(1: 1000) .{ }^{8}$ CF-Correctors were obtained from the Cystic Fibrosis Foundation (USA).

\section{Pulse Chase Labeling}

HEK-293 cells transiently transfected with WT- or S1118F-CFTR cDNA were incubated in MEM medium (Invitrogen) without methionine and cysteine for $60 \mathrm{~min}$ at $37^{\circ} \mathrm{C}$. Proteins being synthesized were then labeled (pulsed) with $0.2 \mathrm{mCi} / \mathrm{ml}{ }^{35} \mathrm{~S}$-Methionine and ${ }^{35} \mathrm{~S}$-Cysteine ( $\operatorname{Tran}^{35} \mathrm{~S}$-Label, MP Biomedicals) for 30 minutes at $37^{\circ} \mathrm{C}$. The plates were washed and incubated without label at $37^{\circ} \mathrm{C}$ for indicated time points (Chase). Then cells were lysed, immunoprecipitated, subjected to SDS-PAGE and blotted for CFTR using the method described above. The radioactivity associated with CFTR was detected using Phosphor Imaging. ${ }^{9}$

\section{Real-time PCR}

RNA was isolated from HEK-293 cells transfected with WT or mutant CFTR using Trizol method (Invitrogen) and its quality was determined to be superior using Nanodrop at Molecular Resource Center at UTHSC (Memphis, TN). cDNA was made using first strand synthesis kit (Invitrogen) and used for real-time PCR (Roche light cycler). Probe designed by Universal ProbeLibrary (Roche) was used and TATA-box binding protein (TBP) was used as the house keeping gene. ${ }^{10}$

\section{lodide Efflux}

HEK-293 cells were transfected with $2 \mu \mathrm{g}$ of pcDNA3 WT-CFTR, pcDNA3 S1118F-CFTR or empty vector (pcDNA3) cDNA. Forty eight (48) hours later iodide efflux was monitored using the previously reported method. ${ }^{11-} 12$ Briefly, cells were loaded for $60 \mathrm{~min}$ at room temperature with loading buffer ( $136 \mathrm{mM} \mathrm{NaI}, 137 \mathrm{mM} \mathrm{NaCl}, 4.5 \mathrm{mM} \mathrm{KH}_{2} \mathrm{PO}_{4}, 1 \mathrm{mM}$ $\mathrm{CaCl}_{2}, 1 \mathrm{mM} \mathrm{MgCl} 2,10 \mathrm{mM}$ glucose, $5 \mathrm{mM}$ HEPES, pH 7.2). Extracellular NaI was washed away thoroughly (7 times) with efflux buffer $\left(136 \mathrm{mM} \mathrm{NaNO}_{3}\right.$ replacing $136 \mathrm{mM} \mathrm{NaI}$ in the loading buffer) and cells were equilibrated for $1 \mathrm{~min}$ in a final 1-ml aliquot. The first four aliquots were used to establish a stable base line in efflux buffer alone. PKA activating agonist cocktail ( $10 \mu \mathrm{M}$ forskolin, $200 \mu \mathrm{M}$ cpt-cAMP and $100 \mu \mathrm{M}$ IBMX) was added to the efflux buffer and samples were collected every minute for $6 \mathrm{~min}$ in the continued presence of agonists (i.e., the efflux buffer used for subsequent replacements also contained agonists at the same concentration). The iodide concentration of each aliquot was determined using an iodidesensitive electrode (Orion Research Inc.) and converted to iodide content.

\section{Sweat Testing}

Diagnostic sweat testing was performed using pilocarpine iontophoresis using duplicate samples from right to left arms. Collection was performed by the filter paper method according to CF Foundation/NCCLS guidelines. ${ }^{13}$ Chloride analysis was performed using a digital chloridometer (Labconco) with a minimal sweat weight of $75 \mathrm{mg}$.

\section{Statistical Analysis}

Statistical significance was obtained using student's $t$-test and $\mathrm{p}$ value less than 0.05 was considered significant $(*)$. 


\section{RESULTS}

\section{An Individual Harboring S1118F-CFTR Exhibits Atypical CF Symptoms}

A Caucasian infant was born at 37 6/7 weeks by cesarean section in Mississippi (USA). The infant's birth weight was $7 \mathrm{lbs} 14$ ounces, Apgar scores were 8 and 9. Prenatal history was remarkable for maternal hypertension and hypothyroidism. At 2 days of age, the infant developed abdominal distension and was transferred to LBCMC. The patient was found to have meconium ileus at exploratory laparotomy and a short segment of bowel resection was preformed and ileostomy placed. The patient was also started on pancreatic enzymes and fat soluble multivitamins for presumed pancreatic insufficiency. Sweat test was performed on two separate occasions at 3 weeks of age which gave normal and intermediate chloride results (22.7, $24.6 \mathrm{mmol} / \mathrm{L} ; 55.1,58.6 \mathrm{mmol} / \mathrm{L})$. Genotyping was sent to Ambry Genetics which showed $\Delta \mathrm{F} 508 / \mathrm{I} 1027 \mathrm{~T}$ on one chromosome and $\mathrm{S} 1118 \mathrm{~F}$ on the other.

The patient did well at home on her diet, enzymes, and vitamins and was subsequently readmitted at 7 weeks of age for surgical closure of the ostomy. Sweat test was repeated at 6 months of age which again gave intermediate chloride results $(55.1,58 \mathrm{mmol} / \mathrm{L})$. The infant's PFTs were normal. Growth parameters were monitored and although rate of weight gain was within normal limits, weight for length measurement remained below the $50^{\text {th }}$ percentile. As part of nutritional assessment at 7 months of age, a fecal elastase test was performed. This result was normal ( $>500 \mathrm{micrograms} / \mathrm{g}$ per Genova) and consistent with pancreatic sufficiency. Pancreatic enzymes were discontinued. Weight and BMI have been followed carefully since then and have increased appropriately.

\section{S1118F-CFTR Makes Less than 10-15\% of Mature CFTR (Band C) Compared to WT-CFTR}

Previous studies in Xenopus oocytes have shown that S1118F-CFTR is a mutant with altered permeation and gating characteristics compared to WT-CFTR. ${ }^{14}$ It is therefore likely that this mutant belongs to Class IV CFTR mutations. However the caveat for this study is that Xenopus oocytes were maintained at $14-18{ }^{\circ} \mathrm{C}$ and therefore interpretation of these results is complicated. Using Quickchange method, we generated S1118F mutation on pcDNA3-CFTR background. We confirmed the sequence and expressed the protein in HEK-293 cells. The cells were grown in DMEM-F12 media (containing 10\% Serum and 1\% Penicillin/Streptomycin) and maintained in $5 \% \mathrm{CO}_{2}$ incubator at $37^{\circ} \mathrm{C}$. CFTR was immunoprecipitated using $\alpha 24-1$ mab (R\& D Systems), subjected to 5\% SDS-PAGE and blotted using the same antibody. The data shows that S1118F-CFTR appears as a $180 \mathrm{kDa}$ immunoreactive band (Band C; the mature CFTR) but is less than 10-15\% of that for WT-CFTR (Fig 1A). No significant amount of the immature CFTR (Band B) was observed for this protein. Iodide efflux measurements were performed using HEK-293 cellstransiently transfected with WT- or S1118F-CFTR cDNA. As shown in Fig. 1B and 1C, S1118F-CFTR is functional but has much lower activity (10-15\%) compared to WT-CFTR. This result is consistent with the protein expression data. In an attempt to define which class this mutation might belong to, we compared S1118F-CFTR with other well-defined classes of CFTR-mutations such as $\triangle$ F508-CFTR (Class II), G551D-CFTR (Class III) and R117H-CFTR (Class IV) and the data is shown in Fig 1 D. It is likely that S1118FCFTR is a trafficking mutant with impaired maturation.

\section{S1118F-CFTR Shows Impaired Maturation}

To test if the reduced expression level of S1118F-CFTR is due to reduced maturation efficiency, we performed pulse chase experiments by treating HEK293 cells expressing S1118F-CFTR or WT-CFTR with a brief pulse for $30 \mathrm{~min}$. The cells were chased for varying time at $37^{\circ} \mathrm{C}$ in the absence of radioactive label, lysed at various time points, mmunoprecipitated using a-24-1 mab and subjected to SDS-PAGE (Fig. 2). The data clearly demonstrates that maturation of CFTR from Band B to Band C is extremely efficient for WT- 
CFTR. However, the maturation is not efficient for S1118F-CFTR and only a small portion of Band $\mathrm{B}$ was converted to band $\mathrm{C}$ (impaired maturation). These biochemical studies are consistent with a mild CF-phenotype observed for individuals harboring S1118F-CFTR as it is a trafficking mutant with impaired maturation efficiency.

To investigate if S1118F-CFTR mRNA has reduced stability, real-time PCR was performed to compare mRNA expression levels in HEK-293 cells transfected with equal amounts of WTor S1118F-CFTR cDNA. The results showed that the mRNA expression level for S1118FCFTR is not reduced compared to WT-CFTR (Fig. 3). It is therefore reasonable to propose that S1118F-CFTR does not belong to the class V mutations.

\section{CF-correctors can Increase the Amount and Function of S1118-CFTR Several Folds}

To test if S1118F-CFTR can be rescued by using CF-correctors, HEK-293 cells expressing WT- or S1118F-CFTR was incubated with CF-corrector C1 [6-(1H-Benzomidazol-2ylsulfanylmethyl)-2-(6-methoxy-4-methyl-quinazolin-2-ylamino)-pyrimidin-4-ol] or C3 [(4cyclohexyloxy-2-\{1-[4-(4-methoxy-benzensulfonyl)-piperazin-1-yl]-ethyl\}-quinazoline], lysed, immunoprecipitated and probed for CFTR. The result showed that treatment of HEK-293 cells expressing S1118F-CFTR with these CF-correctors did increase the mature form of CFTR. In the presence of the CF-Corrector C3, the mature band C increased almost 4-fold (Fig 4A). It is to be noted that cells expressing WT-CFTR were used as a control and a slight increase in the mature band $\mathrm{C}$ was also observed.

Iodide efflux experiments were performed by using HEK-293 cells expressing S1118F-CFTR. As shown in Fig. 4B and 4C, in the presence of CF-Corrector C3, the function of S1118FCFTR increases 2-4 fold. This result is consistent with the Western Blotting result suggesting that S1118F-CFTR mutant can be functionally rescued by using CF-correctors.

\section{DISCUSSION}

S1118F-CFTR is a serine-to-phenylalanine mutation in the $11^{\text {th }}$ transmembrane-spanning domain (TMD) of CFTR. The mutation is cause by a nucleotide change from Cytosine (C) to Thymine (T) at 3485 on CFTR gene and was originally found in a Czech CF patient by Férec C. ${ }^{15}$

To our knowledge, very little work has been done to characterize this CFTR mutant either clinically or at molecular level. Zhang et al expressed S1118F-CFTR mutant in stage V Xenopus oocytes and studied the channel properties electrophysiologically. They found that the mutation affects both permeation and gating properties of the channel. i.e., the open-channel burst duration decreases 10 -fold and the mutant currents are blocked by diphenylamine-2carboxylate (DPC) in a reduced voltage-dependent manner compared to WT-CFTR channel. Based on these observations, they suggested that TMD11 plays a pore-lining role in the functional CFTR channel. ${ }^{14}$

We present here a clinical case in which a patient harboring S1118F-CFTR on one chromosome and $\Delta \mathrm{F} 508 / \mathrm{I} 1027 \mathrm{~T}$ on the other exhibits atypical CF symptoms. The patient developed abdominal distention and was found to have meconium ileus at exploratory laparotomy. Yet, sweat test performed on three separate occasions gave normal to intermediate $\mathrm{Cl}^{-}$levels (22.7, $24.6 \mathrm{mmol} / \mathrm{L} ; 55.1,58.6 \mathrm{mmol} / \mathrm{L}$ and $55.1,58 \mathrm{mmol} / \mathrm{L}$ ). Fecal elastase test at $7 \mathrm{month}$ of age was normal (>500 micrograms/g per Genova) suggesting pancreatic sufficiency.

To help understand this mild CF-phenotype, we used various biochemical methods to characterize S1118F-CFTR mutant at protein level. First, we generated point mutation at position S1118 to phenylalanine (F), confirmed the sequence and expressed the plasmid in 
HEK-293 cell line. Western blotting result showed this mutant only constitutes 10-15\% of mature form CFTR (Band C) compared to WT-CFTR (Fig.1A), suggesting it might be a trafficking mutant with impaired maturation. It is to be noted that $\triangle$ F508-CFTR appeared as an immature form (Band B). Secondly, to investigate if S1118-CFTR is functional (as a $\mathrm{Cl}^{-}$ channel), we performed iodide efflux experiments. The result showed S1118-CFTR is functional but has much lower activity compared to WT-CFTR (Fig. 1B and 1C). This functional result is consistent with the protein expression data and with Zhang's result which showed S1118-CFTR is a functional $\mathrm{Cl}^{-}$channel yet with altered permeation and gating characteristics compared to WT-CFTR. ${ }^{14}$

Thirdly, we compared S1118F-CFTR with other well defined CF-mutations using Western blotting. G551D-CFTR (Class III; regulation mutant) and R117H (Class IV; permeation mutant) are mutations with partial loss of channel function. Based on the observations as described above and Zhang's result, ${ }^{14}$ it is therefore reasonable to assume that S1118F-CFTR might be a trafficking mutant with impaired maturation. Pulse chase experiments were employed to test the maturation efficiency of S1118F-CFTR. The result showed that, in contrast to WT-CFTR, only a small portion of Band B (immature band) was converted to Band C (mature band) confirming impaired maturation. Next, to investigate if the impaired maturation is due to the reduced mRNA stability, we performed real-time PCR to compare mRNA expression levels in HEK-293 cells transfected with equal amounts of WT- or S1118F-CFTR cDNA. The results showed that the mRNA expression level for S1118F-CFTR is not reduced compared to WT-CFTR suggesting that S1118F-CFTR does not belong to class V mutations (Fig. 3).

Class II mutations are mostly processing mutations which are retained in the ER and fail to mature. $\triangle$ F508-CFTR is the most prevalent class II mutant. It has been reported that most of $\triangle$ F508-CFTR get trapped in the ER and is rapidly degraded. $16^{-18}$ Therapy for processing defects mutations (such as $\triangle$ F508-CFTR) would be to find ways to increase the level of functional protein at the plasma membrane. The use of pharmacological chaperones (CFcorrectors) to promote the maturation of such mutants is one of the promising approaches. ${ }^{19-}$ 22 To test if the maturation efficiency for S1118F-CFTR can be improved by using CFcorrectors, we treated HEK-293 cells expressing S1118F-CFTR with CF-correctors C1 and C3. The result showed CF-correctors did increase the maturation efficiency. In the presence of CF-corrector $\mathrm{C} 3$, the mature band $\mathrm{C}$ increased by 3 to 4 fold over untreated cells and the function of S1118F-CFTR increased 2-4 fold as well in iodide efflux experiments.

Consistent with a mild CF-phenotype observed for a patient, our studies suggest that S1118FCFTR is likely to be trafficking mutant with impaired maturation. Moreover, it can be functionally rescued by using CF-correctors currently under development.

To our knowledge, this is the first study in which S1118F-CFTR mutant was fully characterized at the protein level. Our studies may also have significant implication for future development of mutation specific therapies aimed at restoring CFTR function.

\section{Acknowledgments}

We would like to thank William L. Taylor, PhD, Director of Molecular Resource Center (UTHSC, Memphis, TN) for help with real-time PCR studies. We are grateful to the Cystic Fibrosis Foundation (USA) for providing the CFcorrectors used in the study. This work was supported in part by NIH funding DK074996 and DK080834 (to APN), Cystic Fibrosis Foundation-USA Center Grant Program (to DCS) and American Heart Association pre-doctoral fellowship (to PH) and Department of Physiology (UTHSC, Memphis) for P.J. Quigley award (to PH). 


\section{REFERENCES}

1. Riordan JR. Assembly of functional CFTR chloride channels. Annu Rev Physiol 2005;67:701-718. [PubMed: 15709975]

2. Welsh, MJ.; Ramsey, BW.; Accurso, F.; Cutting, GR. Cystic fibrosis. In: Scriver, CL.; Beaudet, AL.; Valle, D.; Sly, WS., editors. The Metabolic and Molecular Basis of Inherited Disease. New York: McGraw-Hill; 2001. p. 5121-5188.

3. Rowntree RK, Harris A. The phenotypic consequences of CFTR mutations. Ann Hum Genet 2003;67:471-485. [PubMed: 12940920]

4. Amaral MD, Kunzelmann K. Molecular targeting of CFTR as a therapeutic approach to cystic fibrosis. Trends Pharmacol Sci 2007;28:334-341. [PubMed: 17573123]

5. Haardt M, Benharouga M, Lechardeur D, Kartner N, Lukacs GL. C-terminal truncations destabilize the cystic fibrosis transmembrane conductance regulator without impairing its biogenesis. A novel class of mutation. J Biol Chem 1999;274:21873-21877. [PubMed: 10419506]

6. Benharouga M, Haardt M, Kartner N, Lukacs GL. COOH-terminal truncations promote proteasomedependent degradation of mature cystic fibrosis transmembrane conductance regulator from postGolgi compartments. J Cell Biol 2001;153:957-970. [PubMed: 11381082]

7. Zielenski J. Genotype and phenotype in cystic fibrosis. Respiration 2000;67:117-133. [PubMed: 10773783]

8. Li C, Dandridge KS, Di A, Marrs KL, Harris EL, Roy K, Jackson JS, Makarova NV, Fujiwara Y, Farrar PL, Nelson DJ, Tigyi GJ, Naren AP. Lysophosphatidic acid inhibits cholera toxin-induced secretory diarrhea through CFTR-dependent protein interactions. J Exp Med 2005;202:975-986. [PubMed: 16203867]

9. Benharouga M, Sharma M, Lukacs GL. CFTR folding and maturation in cells. Methods Mol Med 2002;70:229-243. [PubMed: 11917526]

10. Universal ProbeLibrary System. Roche Applied Science. (https://www.roche-applied-sience.com/sis/rtpcr/upl/index.jsp)

11. Li C, Roy K, Dandridge KS, Naren AP. Molecular assembly of cystic fibrosis transmembrane conductance regulator in plasma membrane. J Biol Chem 2004;279:24673-24684. [PubMed: 15060073]

12. Chang XB, Tabcharani JA, Hou YX, Jensen TJ, Kartner N, Alon N, Hanrahan JW, Riordan JR. Protein kinase A (PKA) still activates CFTR chloride channel after mutagenesis of all 10 PKA consensus phosphorylation sites. J Biol Chem 1993;268:11304-11311. [PubMed: 7684377]

13. NCCLS document C34-A2. Wayne (PA): NCCLS; 2000. Sweat Testing: Sample Collection and Quantitative Analysis; Approved Guideline-Second Edition.

14. Zhang Z, McDonough SI, McCarty NA. Interaction between permeation and gating in a putative pore domain mutant in the cystic fibrosis transmembrane conductance regulator. Biophys J 2000;79:298313. [PubMed: 10866956]

15. Mutation Details for S1118F-CFTR. Cystic Fibrosis Mutation Database. (http://www3.genet.sickkids.on.ca/cftr/MutationDetailPage.external?sp=897).

16. Cheng SH, Gregory RJ, Marshall J, Paul S, Souza DW, White GA, O'Riordan CR, Smith AE. Defective intracellular transport and processing of CFTR is the molecular basis of most cystic fibrosis. Cell 1990;63:827-834. [PubMed: 1699669]

17. Bronsveld I, Mekus F, Bijman J, Ballmann M, de Jonge HR, Laabs U, Halley DJ, Ellemunter H, Mastella G, Thomas S, Veeze HJ, Tümmler B. Chloride conductance and genetic background modulate the cystic fibrosis phenotype of Delta F508 homozygous twins and siblings. J Clin Invest 2001;108:1705-1715. [PubMed: 11733566]

18. Kälin N, Claass A, Sommer M, Puchelle E, Tümmler B. DeltaF508 CFTR protein expression in tissues from patients with cystic fibrosis. J Clin Invest 1999;103:1379-1389. [PubMed: 10330420]

19. Wang Y, Loo TW, Bartlett MC, Clarke DM. Additive effect of multiple pharmacological chaperones on maturation of CFTR processing mutants. Biochem J 2007;406:257-263. [PubMed: 17535157]

20. Wang Y, Bartlett MC, Loo TW, Clarke DM. Specific rescue of cystic fibrosis transmembrane conductance regulator processing mutants using pharmacological chaperones. Mol Pharmacol 2006;70:297-302. [PubMed: 16624886] 
21. Wang Y, Loo TW, Bartlett MC, Clarke DM. Correctors promote maturation of cystic fibrosis transmembrane conductance regulator (CFTR)-processing mutants by binding to the protein. J Biol Chem 2007;282:33247-33251. [PubMed: 17911111]

22. Van Goor F, Straley KS, Cao D, González J, Hadida S, Hazlewood A, Joubran J, Knapp T, Makings LR, Miller M, Neuberger T, Olson E, Panchenko V, Rader J, Singh A, Stack JH, Tung R, Grootenhuis PD, Negulescu P. Rescue of DeltaF508-CFTR trafficking and gating in human cystic fibrosis airway primary cultures by small molecules. Am J Physiol Lung Cell Mol Physiol 2006;290:L1117-L1130. [PubMed: 16443646] 

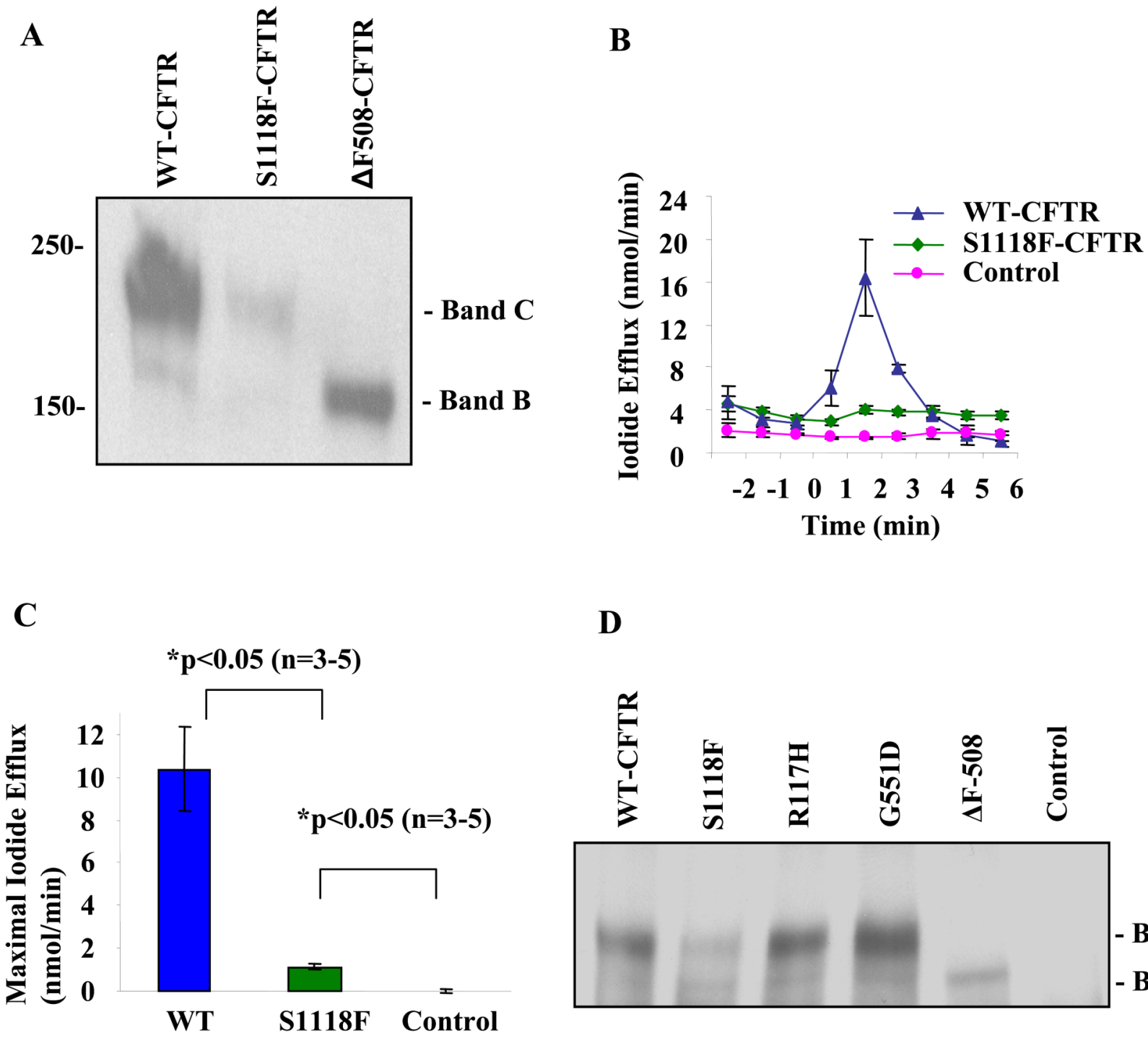

D

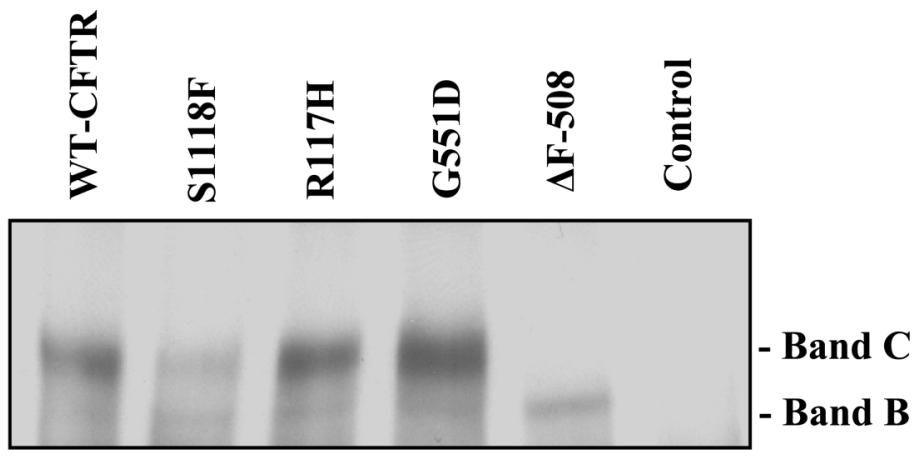

Fig. 1. Characterization of S1118F-CFTR

(A) HEK-293 cells were transiently transfected with pcDNA3 containing WT-, S1118F- or $\triangle$ F508-CFTR cDNA, lysed after 48 hours, immunoprecipitated and western blotted for CFTR ( $\alpha$ 24-1 mab). The experiments were performed 4 times. (B) Iodide efflux assay in HEK-293 cells transiently transfected with WT- or S1118F- CFTR cDNA. PKA activating agonist cocktail (containing 10 $\mu \mathrm{M}$ forskolin, $100 \mu \mathrm{M}$ IBMX and $200 \mu \mathrm{M}$ cpt-cAMP) was added to activate CFTR. Line graph is Iodide efflux (nmol/min) as mean of 3 (WT and Control) or 5 (S1118F-CFTR) experiments with standard error mean (SEM). (C) Bar graph is maximal Iodide efflux at 2 min after adding the agonist cocktail as mean of 3-5 experiments with SEM (calculated by subtracting efflux data at $1 \mathrm{~min}$ from that at $2 \mathrm{~min}$ ). (D) HEK-293 cells were transiently transfected with pCDNA3 containing WT-, S1118F-, R117H-, G551D- or $\Delta$ F508CFTR cDNA, lysed 48 hours later, immunoprecipitated ( $\alpha$ 24-1 mab) and western blotted for CFTR ( $\alpha$ 24-1 mab). The experiments were performed 4 times. 
A

WT-CFTR

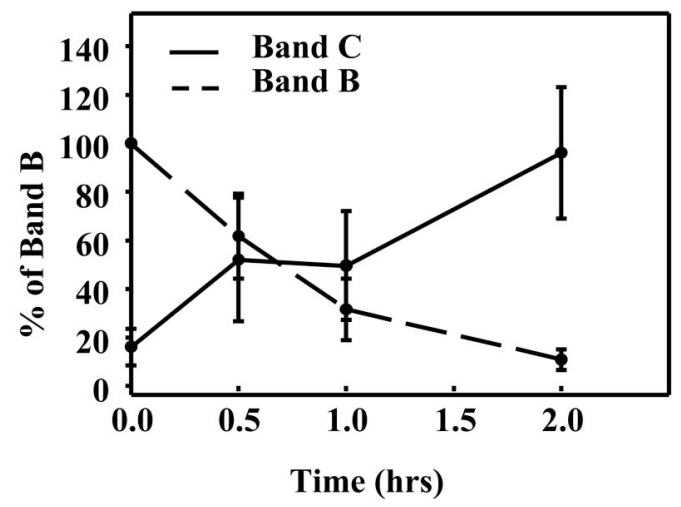

$\begin{array}{llll}0 & 0.5 & 1 & 2\end{array}$

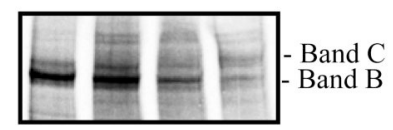

S1118F

B

WT

S1118F-CFTR
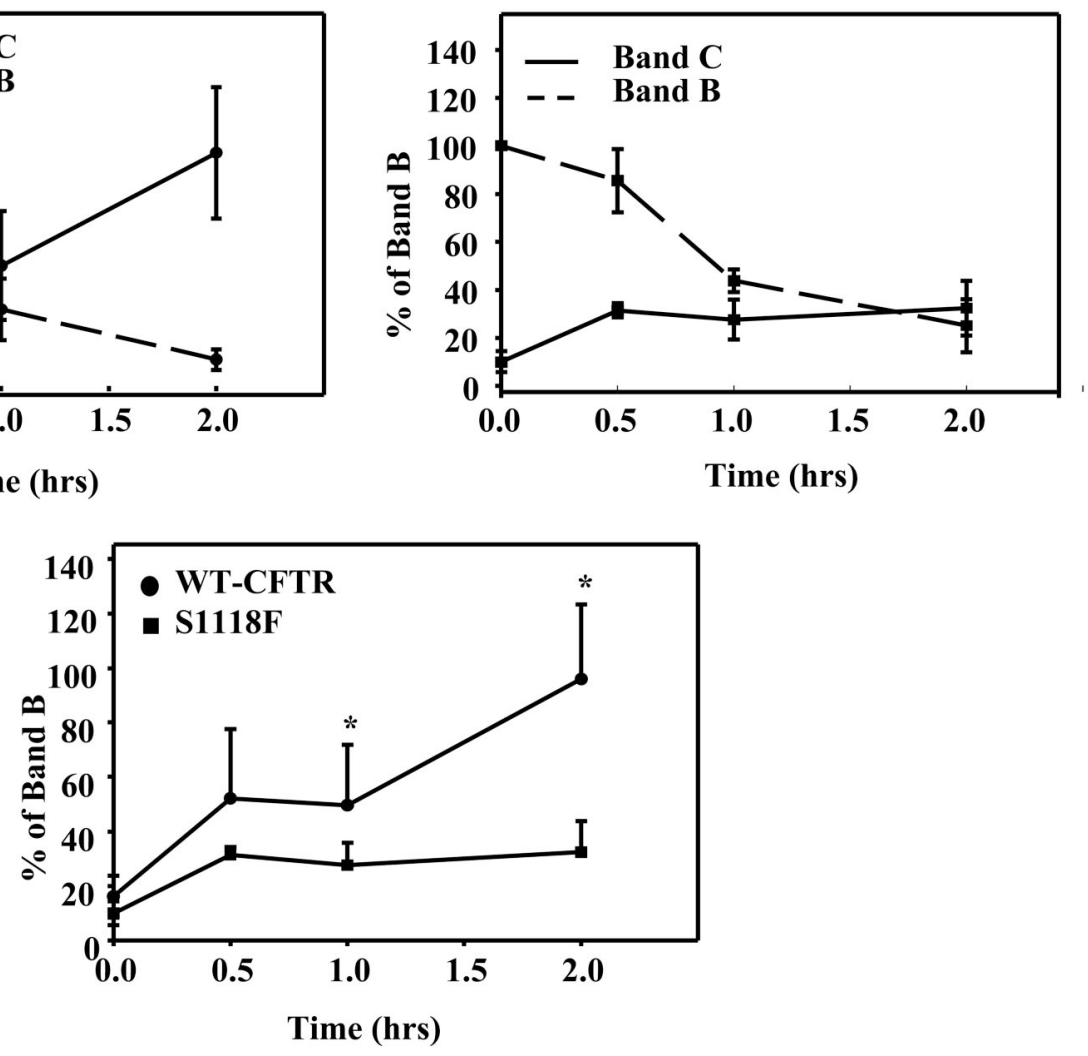

Fig. 2. S1118F-CFTR shows impaired maturation

(A) Pulse-chase experiments to monitor the formation of mature protein over a period of 2 hours in HEK-293 cells transfected with WT-or S1118F-CFTR cDNA. The experiments were performed 4 times. (B) Quantitation of mature (Band C) and immature (Band B) protein in WT- and S1118F-CFTR from pulse-chase experiment showing impaired maturation of S1118F-CFTR. The Band B at time '0' was considered as 100\% for WT- and S1118F-CFTR, respectively. 


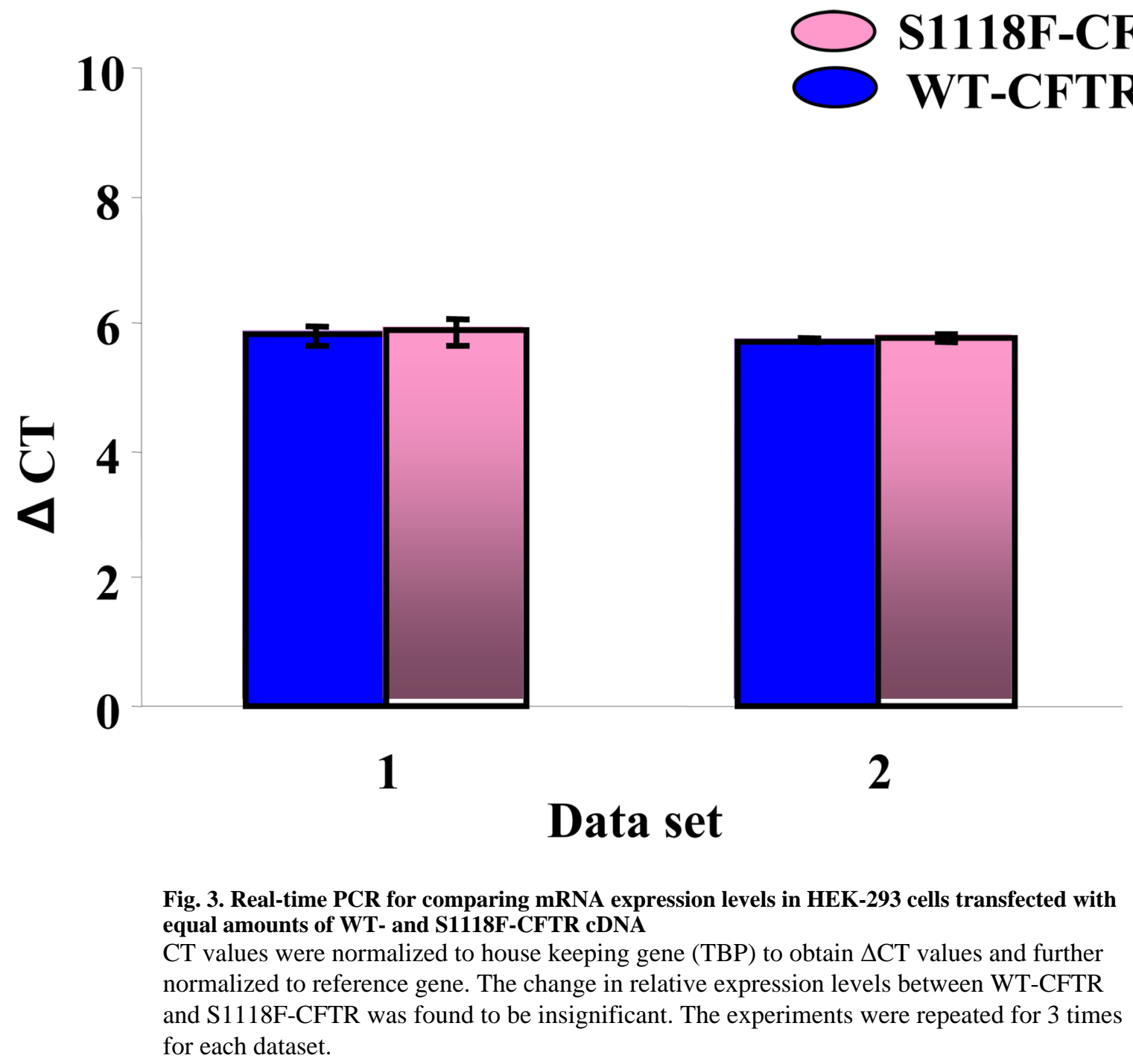


A

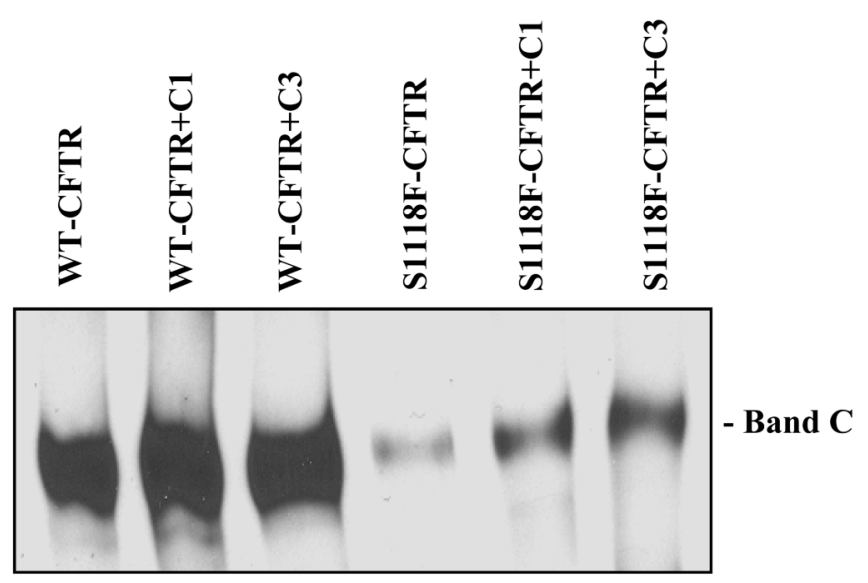

B

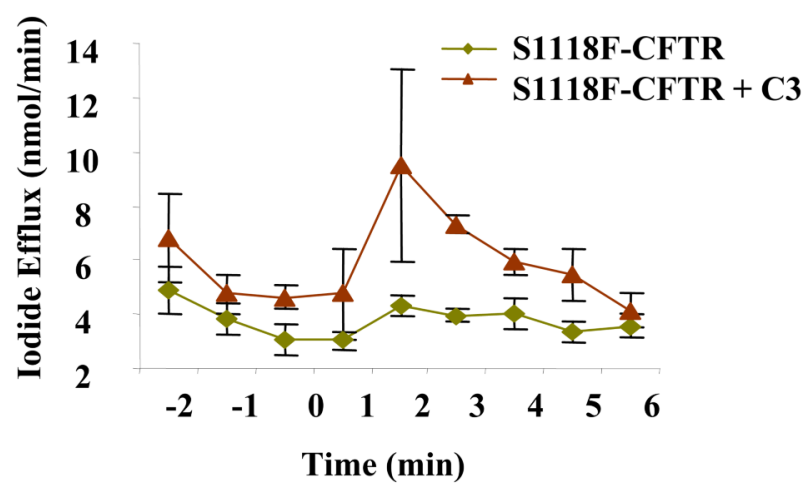

C

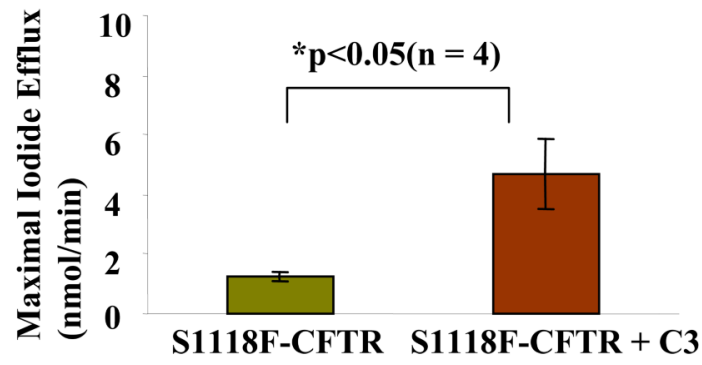

Fig. 4. CF-correctors can increase the amount and function of S1118F-CFTR

(A) HEK-293 cells were transiently transfected with WT- or mutant CFTR cDNA, treated for 16 hours at $37^{\circ} \mathrm{C}$ with $5 \mu \mathrm{M} \mathrm{CF}$ correctors $\mathrm{C} 1$ or $\mathrm{C} 3$, lysed, immunoprecipitated and probed for CFTR as before. The experiments were repeated for 4 times. (B) Iodide efflux assay in HEK-293 cells transiently transfected with S1118F-CFTR with or without CF-corrector C3

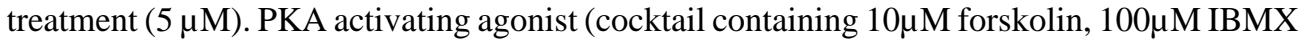
and $200 \mu \mathrm{M}$ cpt-cAMP) was used to activate CFTR. Line graph is Iodide efflux (nmol/min) as mean of 4 experiments with standard error mean (SEM). (C) Bar graph is maximal Iodide efflux at 2 min after adding the agonist cocktail as mean of 4 experiments with SEM (calculated by subtracting efflux data at $1 \mathrm{~min}$ from that at $2 \mathrm{~min}$ ). 